\title{
Online class sa bagong kadawyan: Isang paglalatag ng mga karanasan
}

Gallega, Myka

Veritas College of Irosin, Philippines (sivilliamk@gmail.com)

Guyala, Catherine

Veritas College of Irosin, Philippines (Catherineolanda01@gmail.com)

Formento, Mark Lester

Veritas College of Irosin, Philippines (marklesterformento3@gmail.com)

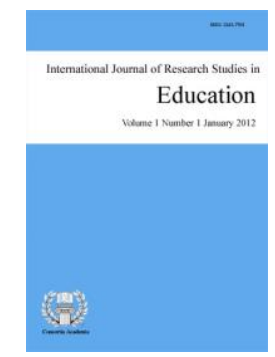

ISSN: 2243-7703 Online ISSN: 2243-7711

OPEN ACCESS

Gamba, Angelo

Veritas College of Irosin, Philippines (Angelogamba98@gmail.com)

Gelilio, Eric

Veritas College of Irosin, Philippines

Sorsogon State University, Philippines

Gallanosa National High School, Philippines (gelilioeric@gmail.com)

\section{Abstract}

Despite of some challenges Gallanosa National High School students, adapt to new learning methods has not been easy for the student, although learning has still continued using the new online class method. Based on the findings of our research, the effectivity and quality of online class is less than the face to face classes and there are chances that the internet connection is weakening. In accordance to our survey, it can be determined that $92 \%$ of the students doesn't have internet connection for online class. Therefore, some schools, together with its stakeholders made a solution to the occurring problem. As for example, the school gave a loaded sim card to the students, helping them to have an internet connection. The discussion about the importance of implementation of online, as an alternative education system in the midst of facing pandemic, explains the importance of online class which implemented during the height of the pandemic period, so that every student does not neglect their studies because of one strong hindrance called COVID-19 pandemic. It also identifies causes and effects to each student such as adverse health on some students, it may be physically, emotionally and mental health, and lack of internet connection and gadgets, which is the most important thing a student needs to have online class. Upon doing our research, we have found out that most of the students prefer to return the former method of teaching-learning because they understand each lesson better.

Keywords: online class proclamation, article, implementation, alternative education strategies, impacts, new normal amid of pandemic, WHO 


\section{Online class sa bagong kadawyan: Isang paglalatag ng mga karanasan}

\section{Introduction}

Ang Edukasyon ay isa sa mga pinakamahalagang aspeto ng buhay ito ang nagbibigay sa atin ng kaalaman na matuto't magtagumpay sa hinaharap ng buhay. Ang edukasyon ay may is ang importante ng layunin na hubugin ng isang bata na maging isang propesyonal paglaki. Ngunits sa ngayon ang pagpapalaganap ng edukasyon ay may salik na kinakaharap, ang pandemya. Sa kagustuhang maipalaganap ang edukasyon sa gitna ng pandemya, ang pamahalaan ay nagpatupad na magkaroon ng tatlong (3) opsyon ng edukasyon, isa na dito ang Distance Learning Class o ang Virtual/Online Class.

Ayon kay Secretary Leonor Briones (2020), ang online learning ang solusyon upang maipagpatuloy ang pag-aaral kahit nasa tahanan. May malaking gampanin ang mga magulang sapagkat sila ang maggagabaysa kanilang mga anak habang wala sa paaralan. Ang distance learning ay naisagawa nang ibang paaralan at maging mga kalapit na bansa. At ito ang solusyon upang maging ligtas ang mga mag-aaral laban sa mga kalamidad at pandemya. Sa panahon ng pandemya, ang online class ang pinakaligtas na pamamaraan ng paghahatid ng edukasyon (Ching at Roberts, 2020; Llaneta, 2021). Subalit sa pagkakaroon ng online class ang may pinakamalaking gampanin sa prosesong ito ay ang mga magulang sapagkat sila ang may kapangyarihan na kontrolin ang kanilang mga anak sa oras ng paggamit ng mga gadgets. Ang mga guro, katuwang ang mga magulang ang magdidisiplina sa kanilang mga anak. Ayon kay Eds Ignacio (2020), nagkakaroon ng disiplina ang mga bata dahil nagkakaroon ng regola sa kanilang tahanan na dapat maglaan ng oras sa paggawa nang aralin, paglalaro o paggamit ng gadgets. At sa kaniya bilang ina, nagging hamon din ang isaayos ang kanyang oras para magampanan ang mga gawaing-bahay at pagsubaybay sa pag-aaral ng anak. Sa kanilang silid ay sadyang naglaan ng study area upang Higit ang konsentrasyon ng kaya napakinabangan ng husto ang study table. Sa pahayag na ito, masusubok ang pasensya't pag-iintindi ng bawat mauling sa kanilang mga anak. Kailangan din gumawa ang mga magulang ng study schedule para magkaroon ng limitasyon ng paggamit ng gadgets para sa ikabubuti ng kalusugan ng kanilang anak.

Ang online class ay isa sa new normal education na ipinapatupad para maipagpatuloy pa rin ang paglaganap ng edukasyon. Mas binibigyan diin at importansya dito ang kahalagahan ng teknolohiya. Ito rin ay paraan ng edukasyon para mahuhubog at magkaroon ng mataas na kalidad ang edukasyon. May pagkakatulad sa face to face na paraan ng pagtuturo at ang makabagong pagtuturo sapagkat makikita at nakakapanayam pa rin nila ang kapwa nila kaklase at mga guro. Ang online class ay idineklara sa (RA 10650) o "Open Distance Learning Law" noong April 12 2020, na naglalayong tumulong sa pagpapalaganap ng edukasyon sa gitna ng pandemya.may iba't-ibang paraan sa paghahatid ng edukasyon sa mga bata. isa na rito ang online class online class sa isa sa mga naging ligtas na paghatid ng kaalaman sa mga estudyante sapagkat hindi lumabas ng kani-kanilang mga tahanan ngunit ang pagkakaroon ng online education ay may mga salik na kinakaharap lalo na sa mga guro at estudyante. Ang isa mga salik o problemang dulot ng online class ay ang mental health ng mga bata. Ayon sa ulat ni Jeffrey Hernaez ng ABS-CBN News noong October 11, 2020, ang mga bata sa kani-kanilang bahay ay nagiging malungkutin at nawawalan na ng gana gumawa ng kanilang mga gawain. Ang online class ay may malaking epekto sa mental health ng mga bata. Kaya kailangan ng mga magulang magkaroon ng positive discipline sa pagdidisiplina ng kanilang bata.

Ayon sa World Health Organization-Philippines, umaabot sa Higit 3 milyong Pilipino ang nakararanas ng mental, neurological at substance use disorder sa unang bahagi pa lamang ng taon. Kaya nagpaalala si Dhanika Garcia, Secretary-General ng Mental Health Ph, na huwag mahiyang kumonsulta kung nakararanas ng problema. Sa pananaliksik ni Joe S. Take, napag-alamang ang implementasyon ng online class ay nagiging sanhi ng pagkakaroon ng dibisyon sa pagitan ng mga mahihirap at ng mga may sapat na pera para matustusan ang mga kagamitang kailangan upang makasabay sa bagong normal na paraan ng pag-aaral. Sa pahayag nyang ito, 
mapapansin na ang mga mag-aaral sa buong Pilipinas ay mahihirapan na matugunan ang mga kakailanganin sa kanilang online class.

Ang online class ay may mabuti’t magandang naiidulot sa pagpapalaganap at paghahatid ng edukasyon sa mga estudyante. Alinsunod sa mga impormasyong nabanggit sa itaas, nailahad dito ang kahalagahan at suliraning kinakaharap ng mga stakeholder's ng mga paaralan lalo na ng mga estudyante. May mga solusyong inihanda ang DepEd, subalit may mga pagkakataon at lugar na ang solusyong ito ay hindi natutugunan. Ito ay sa kadahilanang malayo o nasa remote area ang mga lokasyon ng mga bata at ang iba ay walang kakayahang makabili ng mga gadgets nakakailanganin sa online class. Bukod sa mga salik sa implementasyon ng online class, may mahalagang dulot din ito sa atin at lalo na sa mga estudyante. Ito ang pinakaligtas na pamamaraan ng paghatid ng edukasyon sa gitna ng pandemya. Dahil dito, makakaiwas ang mga kabataan sa COVID-19 dahil hindi na nila kailangan lumabas. Ang online class ay para na ring itong face to face, makikita't makakausap pa rin ang guro't kaklase.

\subsection{Paglalahad ng Suliranin}

Ang pananaliksik na ito ay nakatampulan sa pangunahing layunin nito na kung saan mailalahad at maipapakita ang kahalagahan at salik sa Online Class sa Bagong Kadawyan. Nilalayon nitong tukuyin ang mga salik at apektado ng online class:

> Ano-ano ang mga salik na nakakaapekto sa pagkatuto ng online class na modalidad sa bagong kadawyan?

$>\quad$ Sa panahon nang kadawayan (new normal) mas marami ang naapektuhan. Unang una na dito ang sistema ng edukasyon. Makabibigay ito ng salik at problema sa kalusugan man at pinansyal. a) mental health, b) physical health, at c) pinansyal

$>\quad$ Ano ang nabuong rekomendasyon batay sa resulta ng pag-aaral?

\section{Pamamaraang ginamit}

Sa pag-aaral na ito, masinop na kinalap ng mga mananaliksik, ang anumang datos na patungkol sa online class sa bagong kadawyan. Gumamit ang mga mananaliksik ng "quantity methods" o ang paraan g kuwantitatib. Ginamit ito sa pagkalap ng bilang ng mga datos mula sa mga kinapanayam. Ang mga mananaliksik ay ginamit ang teknolohiya sa pakikipanayam sa mga estudyante ng Gallanosa National High School. Ginamit nila ang Messenger App sa pakikipagpanayam sa mga estudyante ng Gallanosa National High School. Ginamit ito para sa kaligtasan at alinsunod na din sa safety Protocol ng World Health Organization WHO. gumamit din ng internet access o connection para sa malawakang pagsisiyasat ukol sa paksang "online Class. Ang mga mananaliksik ay gumamit ng Systematic Random sampling sa pag-aanalisa kung ilang katao lang ang pweding kapanayamin.

\section{Mga Natuklasan}

Inilalahad sa kabanatang ito ay interpretasyon ng mga nakalap na datos at natukoy ang epekto ng online class sa mga mag-aaral ng Gallanosa National Highschool. Ang bilang ng mga respondante ay 100 na nag mula sa babae at lalaki. May edad na 16 hanggang 19 taong gulang at nasa 11-12 grado.

\section{Talahanayan 1}

May kakayahan sa paggamit ng internet

\begin{tabular}{lcc}
\hline \multicolumn{1}{c}{ Nakakagamit ng internet } & Bilang ng sumagot & Bahagdan \\
\hline Nakakagamit ng internet & 8 & $8 \%$ \\
Hindi nakakagamit ng internet & 92 & $92 \%$ \\
\hline
\end{tabular}

Sa talahanayan na ito makikita na kakaunti lamang ang may kakayahan na makagamit ng internet para sa 
Gallega, M., Guyala, C., Formento, M. L., Gamba, A., \& Gelilio, E.

kanilang online class. May roon 8 nabilang o $8 \%$ na bahagdan ang sumagot na sa palagay nila ay mayroon pagkakataon na makagamit ng internet at 92 na bilang o $92 \%$ na bahagdan ang nag sabi na hindi lahat at may roong internet para sa online class.

Ayon kay Knowles (2005), ang online learning ay mayroong hindi magagandag resulta sa mga mag-aaral tulad na lamang ng pagkakaroon ng mahinang internet connection habang nagkaklase o kaya naman ay hindi pagkaintindi ng mahusay dahil sa iba't ibang persepyon at pag-unawa ng mga mag-aaral. Ang ibang mag-aaral ay hindi nakakagamit ng internet sa kanilang online class.

\section{Talahanayan 2}

Epektibo o mabisa ba ang online class

\begin{tabular}{lcc}
\hline \multicolumn{1}{c}{ Epektibo o Mabisa } & Bilang ng sumagot & Bahagdan \\
\hline Epektibo ang online class & 64 & $64 \%$ \\
Hindi epektibo ang online class & 36 & $36 \%$ \\
\hline
\end{tabular}

Makikita sa talahanayang ito na 64 na bahagdan ang nag sabi na epektibo at mabisa ang online class sa bagong kadawyan at may roong 36 bahagdan naman ang nagsabing hindi ito epektibo para sa kanila. Ayon kay Grace Ramos, dating guro sa Pilipinas, di gaanong epektibo ang online class lalo at may mga pagkakataon na humihina ang internet connection. At iba pa rin ang personal na interaksyon sa pagitan ng guro at mga mag-aaral at pagkakaroonng aktibidad na magkakasama ang mga kaklase.

\section{Talahanayan 3}

Mga apektado ng Online class

\begin{tabular}{lcc}
\hline \multicolumn{1}{c}{ Mga apektado ng Online Class } & Bilang ng sumagot & Bahagdan \\
\hline Mental health at Physical Health & 84 & $84 \%$ \\
Pinansyal & 16 & $16 \%$ \\
\hline
\end{tabular}

Ayon sa datos na ito may roong 84 na bahagdan sa survey ang magsasabing mayroon masamang epekto sa kanila ang online class. Tulad ng stress at pagkamainitin ng ulo. Subalit mayroon namang 16 bahagdan lamang ang nagsabi na hindi nito na apektohan ang kanilang kalusugan. Ayon sa tala ng philstar.com, ang online learning ay nagreresulta sa mental stress sa mga bata na nawalan ng oportunidad na makipagkaibigan o makipaglaro sa kapwa bata.

\section{Talahanayan 4}

Nasisiyahan ang mga mag aaral sa online class sa bagong kadawyan

\begin{tabular}{lcc}
\hline & Bilang ng sumagot & Bahagdan \\
\hline $\begin{array}{l}\text { Estudyanteng Nasisiyahan sa online } \\
\text { class. }\end{array}$ & 94 & $31 \%$ \\
$\begin{array}{l}\text { Estudyanteng HINDI Nasisiyahan sa } \\
\text { online class }\end{array}$ & 206 & $69 \%$ \\
\hline
\end{tabular}

Sa talahanayang ito may roong tatlong tanong na pinagsama sapagkat mayroon ito magkakatulad na pakahulugan. Mayroong 300 na sagot sa bawat tanong. Sa kabuoan may roong 206 na bilang o $68 \%$ na bahagdan ang suamagot na hindi nila gusto o sila ay hindi nasisiyahan sa online class ng bagong kadawyan at myroong lamang 94 na bilang o $31 \%$ na bahagdan ang nagsabi na sila ay nasisiyahan o gusto nila ang online class ng bagong kadawyan.

Ayon sa kingjamesalmirol blogspot (2020), bilang estudyante na nakakaranas ng online class masasabi kong may magandang epekto din ito dahil sa simula ng nangyari ito ay hindi ko na kailangan pang gumising ng ganoon kaaga kumpara sa normal na pasok at mas nabibigyan ako ng mahabang oras na tapusin ang mga aktibidad at gawain na ibinababa sa amin. Dahil din dito ay mas magiging maayos ang aking gawain dahil nagagabayan ako ng aking mga magulang at kapatid sa paggawa ng mga gawain. 


\section{Talahanayan 5}

May natututunan ba ang mga mag-aaral sa online class

\begin{tabular}{ccc}
\hline \multicolumn{1}{c}{ May natutunan } & Bilang ng sumagot & Bahagdan \\
\hline May natututunan sa online class & 22 & $22 \%$ \\
Walang natututunan sa online class & 78 & $78 \%$ \\
\hline
\end{tabular}

Sa talahanayang ito makikita ang datos ng sagot ng mga mag aaral kung mayroon ba silang natututunan sa kanilang online class. Mayroong 22 na bilang o 22 bahagdan lamang ang may sabing mayroon silang natututunan sa kanilang asignatura sa online class, at mayroon namang 78 na bilang o 78 na bahagdan ang nag sasabing wala silang natututunan sa kanilang asignatura sa online class. Mayroong 150 na respondent sa pananaliksik na ito.

Ayon sa tala ng PressReader.com, wala pang 45 porsyento sa kanila ang nagsabing magulang na natuto ang kanilang mga anak sa distance learning. Ibig sabihin, duda sila na mayroong natutunan ang mga anak sa bagong sistema ng pag-aaral. Maraming nga nagulang at mag-aaral na nahihirapang intindihin ang mga self-learning modules kasi parang "self-study" lamang ang kalalabasan.

\section{Talahanayan 6}

Ano ang mas pipiliin mo? Face to face o online class?

\begin{tabular}{lcc}
\hline & Bilang ng sumagot & Bahagdan \\
\hline Face to face & 91 & $91 \%$ \\
Online class & 9 & $9 \%$ \\
\hline
\end{tabular}

Sa talahanayang ito makikita ang datos kung ano ang mas pinili ng mga studyante sa kanilang pag-aaral. May kabuoan na 100 na respondent sa tanong na ito. May 91 na bilang o 91 na bahagdan ang pinili na magkaroon na muli ng Face to face classes. Samantalang mayroon lamang 9 na bilang o 9 na bahagdan ang gustong ipagpatuloy. Ayon sa mga sagot sa brainly.com, karamihan sa mga estudyante ay mas gusto ang face to face dahil mas natututo sila dito sa pamamaraang ito.

\subsection{Impak ng Online Class sa mga sumusunod na aspeto ng pagkatuto}

Batay sa mga impormasyon mula sa tagatugon ang Online Class ay may positibo at negatibong impak sa buhay estudyante. Ang mga tagatugon may iba’t ibang sagot sa mga impak ng online class. Ayon kay Pastor Joey Umali noong September 25, 2021, ang online class ay mayroong epekto sa mental, pisikal at pinansyal na aspeto ng buhay ng mga estudyante.

\subsection{Mental Health}

\section{Talahanayan 7}

Mga Positibong impak ng Online Class sa mga estudyante sa makabagong kadawyan

\begin{tabular}{lcc}
\hline \multicolumn{1}{c}{ Positibong Impak } & Bilang ng mga Sumagot & Bahagdan \\
\hline $\begin{array}{l}\text { Naipagpapatuloy ang pag-aaral at nadadagdagan ang } \\
\text { kaalaman. }\end{array}$ & 14 & 9.33 \\
$\begin{array}{l}\text { Sinasanay ang mga mag-aaral na maging responsible at } \\
\text { independent }\end{array}$ & 38 & 25.33 \\
$\begin{array}{l}\text { Ang pagkayamot at lungkot ng mga bata ay nawawala dahil } \\
\text { mayroon na silang libangan. }\end{array}$ & 20 & 13.33 \\
$\begin{array}{l}\text { Mas napapalawak ang kanilang imahinasyon sa mga } \\
\text { aktibidad na nagmumula sa kanilang modyul. }\end{array}$ & 39 & 26 \\
$\begin{array}{l}\text { Pinapalakas nito ang pang-unawa at kritikal na pagtatasa } \\
\text { ng isip ng mga kabataan. }\end{array}$ & 33 & 22 \\
\hline
\end{tabular}

Alinsunod sa mga datos na nakalap, ang talahayang ito ay nagbibigay impormasyong ukol sa positibong impak ng online class sa mga estudyante. Ang mga mag-aaral ay nakapagbigay ng pare-parehong sagot sa katanungang ukol sa positibong impak. Makikita sa mga datos na nakalap na ang online class ay nagpapalawak 
Gallega, M., Guyala, C., Formento, M. L., Gamba, A., \& Gelilio, E.

at nagpapatibay ng kritikal at kahusayan sa pagbuo ng ideya at imahinasyon.

\section{Talahanayan 8}

Negatibong impak ng o Online Class sa mga estudyante ng makabagong Kadawyan

\begin{tabular}{lcc}
\hline \multicolumn{1}{c}{ Negatibong Impak } & Bilang ng Sumagot & Bahagdan \\
\hline Nakakastress at nakakapressure ang online class na & 78 & 52 \\
maaaring magdulot ng depression at pagsuicide. & 28 & 18.67 \\
Nakakawalang gana mag-aral. & 30 & 20 \\
Di maintindihan ang module o ang aralin & 14 & 9.33 \\
Pagiging lutang ng mga estudyante dahil sa pag-iisip ng & 6 & 4 \\
isasagot. & & 4 \\
Nagdudulot ng pagiging mahiyain ng estudyante. & & \\
\hline
\end{tabular}

Ang talahayang ito, makikita ang mga datos ukol sa mga negatibong impak sa kalusugang kaisipan ng mga estudyante na dulot ng Online Class. Matutukoy sa mga datos na malaki ang negatibong impak ng online class sa isip ng mga estudyantye. Halos kalahating porsyento nito ay tungkol sa stress impak na maaaring maging sanhi ng pagkitil ng buhay ng isang estudyante. Ayon sa Nikahku, isang wallpaper ideas website, ang isang halimbawa ng negatibong impak ay ang 14 anyos na dalagita ang nagsuicide dahil sa labis na pressure na ibinibigay ng kanyang magulang sa pagsasagot ng modyul.

\subsection{Physical Health}

\section{Talahanayan 9}

Positibong Impak ng Online Class sa Physical Health ng mga estudyante ng makabagong Kadawyan

\begin{tabular}{lcc}
\hline \multicolumn{1}{c}{ Positibong impak } & Bilang ng Sumagot & Bahagdan \\
\hline $\begin{array}{l}\text { Nakakapagpahinga at relax pakatapos ng module. } \\
\text { Nakakaiwas sa sakit na lumalaganap ngayon o mas kilala bilang }\end{array}$ & 23 & 32.67 \\
$\begin{array}{l}\text { COVID-19 } \\
\text { Hindi na mapapagod sa biyahe ang mga estudyante pagpasok at } \\
\text { pag-uwi sa paaralan at bahay. }\end{array}$ & 19 & 12.67 \\
$\begin{array}{l}\text { Mas napaghuhusayan at nahahasa ang talento sa paggamit at } \\
\text { manipula ng teknolohiya. }\end{array}$ & 26 & 17.33 \\
$\begin{array}{l}\text { Nahuhubog at natutklasan ang iba pang talento dahil mas marami } \\
\text { na ang oras sa bahay kesa sa paaralan. } \\
\begin{array}{l}\text { May sapat na oras na makatulong sa magulang sa mga gawaing } \\
\text { bahay. }\end{array}\end{array}$ & 21 & 14 \\
\hline
\end{tabular}

Sa talahayang ito, naihahayag ang mga positibong impak ng Online Class sa kalusugang pisikal ng mga estudyante sa panahon ng pandemya. Ayon sa karamihang sagot ng estudyante, dahil sa online class mas nagiging ligtas ang mga estudyante sa banta ng COVID-19. Karamihan din nagsasabi na ang mga online class ay nagging paraan upang makatulong sa magulang sa mga gawaing bahay.

\section{Talahanayan 10}

Positibong Impakng Online Class sa Physical Health ng mga Estudyante sa Makabagong Kadawyan

\begin{tabular}{lcc}
\hline \multicolumn{1}{c}{ Negatibong Impak } & Bilang ng mga Sumagot & Bahagdan \\
\hline $\begin{array}{l}\text { Unti-unting panlalabo ng mata dahil sa radiation ng gadgets sa } \\
\text { maghapong pagkakatutok dito. }\end{array}$ & 32 & 21.33 \\
$\begin{array}{l}\text { Nagiging matamlayin at sakitin dahil sa kakulangan sa pisikal na } \\
\text { aktibidad. }\end{array}$ & 22 & 14.67 \\
$\begin{array}{l}\text { Kawalan ng ganang kumain. } \\
\text { Nagkakaroon ng problema sa ergonomika na kung saan ang mga }\end{array}$ & 29 & 19.33 \\
$\begin{array}{l}\text { estudyante ay may posibilidad na magkaroon ng pisikal na } \\
\text { disablidad tulad ng scoliosis , bunga ng maghapong nakaupo sa }\end{array}$ & & 10 \\
$\begin{array}{l}\text { harap ng computer at modyul. } \\
\text { Pangangayayat at pagiging matamlay dahil kakulangan sa tulog sa } \\
\text { kakaisip kung ano ang isasagot sa modyul. }\end{array}$ & 52 & 34.67 \\
\hline
\end{tabular}

Alinsunod sa mga impormasyon o datos na nakalap ng aming grupo, makikita sa talahayang ito ang 
masamang dulot ng online class sa kalusugang pisikal ng mga estudyante. Dahil nga apektado ng online class ang isip ng isang tao, nadadamay nito ang pisikal na kalusugan ng tao. Sa impormasyong nakalap, ang pangangayayat ng mga estudyante ay bunga ng Online Class. Sapagkat hindi na nagkakaroon ng aktibidad na kaugnay sa pangangalaga ng kalusugan at napapagod na din ang mga kabataan sa pag-iisip kung ano ang isasagot sa modyul.

Ayon kay Wiles (2020), ang pagsasagawang mga mga klase sa Online ay talagang nakakaubos dahil dito ay nangangailangan ng mas lakas dito ay nangangailangan ng mas maraming trabaho upang lubos na maintindihan ang mga aralin. Ipinaliwang din sa isang artikulo sa Naional Geographic na ang pagtutok sa online gaya ng mga video call ay nangangailangan ng matinding atensyon na nagreresulta sa pagkapagod ng pangkalahatang kalusugan. Ang pinakaapektado din ng online class ay ang mata ng mga estudyante, dahil nga sa maghapong nakatutok sila sa gadget, tulad ng computer, mas matinding radiation ang nakakaapekto sa kanilang mata.

\subsection{Pinansyal}

\section{Talahanayan 11}

Positibong Impakng Online Class sa aspektong Pinansyal sa Gitna ng Pandemya

\begin{tabular}{lcc}
\hline \multicolumn{1}{c}{ Positibong Impak } & Bilang ng mga Sumagot & Bahagdan \\
\hline $\begin{array}{l}\text { Mas makakatipid sa pamasahe dahil } \\
\text { hindi na kailangang pumunta sa } \\
\text { paaralan sa pag-attend ng klase. }\end{array}$ & 31 & 20.67 \\
$\begin{array}{l}\text { Nagkakaroon ng oras ang ibang } \\
\text { kabataan na magkaroon ng raket tulad } \\
\text { ng online selling,itbpa. }\end{array}$ & 62 & 41.33 \\
$\begin{array}{l}\text { Mas nakakatipid ang mga estudyante sa } \\
\text { mga output at araw-araw na ambagan }\end{array}$ & 11 & 7.33 \\
$\begin{array}{l}\text { sa paaralan. } \\
\text { Nakakapag-ipon ang mga kabataan sa } \\
\text { perang binibigay sa kanila. } \\
\text { Ang internet data na mula sa libreng } \\
\text { sim card mula sa paaralan ay } \\
\text { nakakatulong sa mga estudyante. }\end{array}$ & 25 & 16.67 \\
\hline
\end{tabular}

Sa talahayang ito, makikita na halos lahat ng sagot ng estudyante ay nagkakaroon sila ng sapat na oras para magkaroon ng raket o pagkakakitaan. Dahil sa teknolohiya, mas lumawak ang kaisipan ng mga mag-aaral na gawing negosyo ang online selling sa impluwensya na din ng online class. Dahil sa online class natututo ang kabataan maging madiskarte sa buhay at makapag-ipon. Sa pamamagitan ng pagbibigay ng libreng sim card ng paaralan sa mga estudyante, hindi na masyadong namomroblema ang kanilang magulang kung saan kukuha ng pangtustus sa pang-internet.

\section{Talahanayan 12}

Negatibong impak ng Online sa aspektong Pinansyal sa Gitna ng Pandemya

\begin{tabular}{lcc}
\hline \multicolumn{1}{c}{ Negatibong Impak } & Bilang ng mga Sumagot & Bahagdan \\
\hline $\begin{array}{l}\text { Mas lalong nakadagdag gastusin ang online class . } \\
\begin{array}{l}\text { Dahil sa kahirapang pinansyal, ang ibang bata ay walang } \\
\text { kakayahang makabili ng gadgets para sa online class. }\end{array}\end{array}$ & 37 & 32.67 \\
$\begin{array}{l}\text { May mga pagkakataong ang signal ay mahina kaya ang } \\
\text { ibang magulang ay nagpapakabit na lang ng internet , na } \\
\text { kung saan mas lalong mahal ang load o bayad nito. }\end{array}$ & 23 & 15.33 \\
$\begin{array}{l}\text { Imbes pangbili na lang ng pagkain, ang gagainn na lang sa } \\
\text { pera ay ipapangload na lang para makapasok lang sa online } \\
\text { class. }\end{array}$ & 23 & 15.33 \\
$\begin{array}{l}\text { Mas doble ang gastos na dulot nito dahil ang ibang output } \\
\text { ay kailangang iprint o idownload. }\end{array}$ & 18 & 12 \\
\hline
\end{tabular}

Ang talahayan na ito ay batay sa mga nakalap na impormasyon tungkol sa impak ng online class sa aspektong pinansyal. Dito napatunayan na malaki ang impak ng online class sa usapang pinansyal. Dahil sa hirap 
Gallega, M., Guyala, C., Formento, M. L., Gamba, A., \& Gelilio, E.

ng buhay, karamihan sa mga magulang ang hindi kayang tugunan ang mga pangangailangan ng kanilang mga anak sa sa usaping pinansyal. Karamihan sa mga estudyanteng may online class ay hindi nakakapasok dahil sa walang load o kaya naman mahina ang signal. Sa pagbubukas at pag-implementa ng online class, mas lalong nakikita ang pagkakalayo ng antasng buhay ng tao. Karamihan sa mga magulang ang walang sapat na panggastos sa pagbili ng gadgets para sa online class. Kaya ang ibang estudyante ay nahinto sa pag-aaral o kaya naman hindi na nakakapag-online class.

\section{Konklusyon}

Batay sa mga nakalap at natuklasan na impormasyon, ito ang nabuong konklusyon:

$>\quad$ Ang bawat mag-aaral ng SHS ng GNHS, ay may iba’t ibang pananaw ukol sa online class maaaring makapagbago ng sistema ng edukasyon sa gitna ng pandemya.

$>\quad$ Ang online class sa gitna ay may mga mabuting naidudulot ngunit may masamang dulot naman ito sa mga mental health ng mag-aaral. Natalakay din sa usaping online class ang mga salik at kalidad ng edukasyon na tinatamasa ngayon ng mga mag-aaral ng SHS ng GNHS.

> Ang mga impormasyong ukol sa online class sa gitna ng pandemya ay may buod na siyang makakapagbibigay ng konklusyong kailangan ng mga mag-aaral ang sistemang face to face ng edukasyon. Base sa mga nakalap na rekomendasyon galing sa mga kapwa naming estudyante mas gugustuhin nilang ipatupad muli ang face to face sapagkat mas malaki, ang ambag ng kaalaman kung malinaw sa kanila ang mga leksyon na itinuturo ng kanilang guro.

> Sa gitna ng pandemya mas mainam na makaroong online class para makaiwas sa nakakahawang sakit. Ito ang pinakaligtas na sistema ng edukasyon ngayon. Kaya sobrang mahalaga ito, may mga salik man ito pero kaya pa rin namang bigyang ng solusyon.

\subsection{Rekomendasyon}

Sa mga mata ng mga mananaliksik, mahalagang malaman ng mga mag- aaral na kailangan pahalagahan an pag aarawal kahit na sa panahon ng pandemya.

$>$ Kailangan parin Ang gabay ng mga magulang pag dating sa pag gamit ng teknolohiya para maiwasan Ang mga pangyayari sa mga bata na hindi kaaya-aya.

D Bigyang control ang mga kabataan sa pag gamit ng teknolohiya para maiwasan ang mga masasamang epekto sa kalusogan.

$>\quad$ Maging mapag matsyag sa mga bagay bagay na nailalabas sa internet para maiwasan ang pag labas ng pronograpiya. Kapag my nakitang link

\section{Talasanggunian}

Acuzar, R., Algar, E., \& Mercene, M. (2021). Kwentong distance learning mga tinig ng estudyante, magulang at guro. Los Banos Times.

https://btimes.ph/2021/06/08/kwentong-distance-learning-mga-tinig-ng-estudyante-magulang-at-guro/

Celso, P. E. C. (2021). [OPINYON] Pandemya, pandaraya, at panawagan, Makati City. Rappler.

https://www.rappler.com/voices/imho/opinion-pandemic-online-cheating-call/

Ching, G. S., \& Roberts, A. (2020). Evaluating the pedagogy of technology integrated teaching and learning: An overview. International Journal of Research Studies in Education, 9(6), 37-50. https://doi.org/10.5861/ijrse.2020.5800

Fernandez, A. G. (2020). Ang distance learning at ang sining ng pagtuturo at pagkatuto ng mga mag-aaral sa 
panahon ng pandemya, Bataan National High School-Junior High School. Retrieved from https://depedbalangacity.com/ang-distance-learning-at-ang-sining-ng-pagtuturo-at-pagkatuto-ng-mga-m ag-aaral-sa-panahon-ng-pandemya/

Gunigundo, M. (2021). Online learning sa new normal ng edukasyon. Retrieved from https://journal.com.ph/online-learning-sa-new-normal-ng-edukasyon/

Guro ako Admin. (2020). New normal education. Online and blended learning. Retrieved from http://guroako.com/2020/05/11/new-normal-education-online-and-blended-learning/

Ignacio, E., \& Ramos, G. (2020). Online class, ang bagong normal na pag-aaral ng mga kabataan. Retrieved from

https://www.akoaypilipino.eu/lifestyle/online-class-ang-bagong-normal-na-pag-aaral-ng-mga-kabataan/

Ikademya Mentors. (2021). Usapang module sa kapihan ng ikademya: Mga Hamon sa pagkatuto sa Panahon ng bagong Kadawyan. Ikademya.

Ki. (2020). Opinyon tungkol sa online class/modular learning. PhilNews. https://philnews.ph/2020/12/30/opinyon-tungkol-sa-online-class-modular-learning/

Llaneta, R. N. (2021). COVID-19 pandemic: Hamon sa kahandaan ng mga irosanong gurong magulang sa paaralan at tahanan. International Journal of Research Studies in Education, 10(4), 79-91. https://doi.org/10.5861/ijrse.2021.622

Magsabol, B. (2020). Student's mental health issue during distance learning due to interplay of factors'-expert. Rappler. Retrieved from https://www.rappler.com/nation/students-mental-health-concerns-distance-learning-due-interplay-factor s/

Montemayor, M. T. (2020). Long online class hours may not be good on Learners' health. DOH.

Perez, A. (2021). Survey ukol sa karanasan sa distance learning isinasagawa ng Deped. ABS-CBN. https://news.abs-cbn.com/news/01/05/21/survey-ukol-sa-karanasan-sa-distance-learning-isinasagawa-n g-deped

Pulmano, J. (2020). Epekto ng online class. Retrieved from https://www.scribd.com/document/487145999/EPEKTO-11-25-20

Relativo, J. (2020). Deped: Huwag lugnay ang student teacher deaths sa distance learning. PhilStar. https://www.philstar.com/pilipino-star-ngayon/bansa/2020/10/20/2050972/deped-huwag-iugnay-ang-stu dent-teacher-deaths-sa-distance-learning

Take, J. S. (2020). Mga pagsubok sa bagong normal sa edukasyon. Pilipino Mirror. https://pilipinomirror.com/mga-pagsubok-sa-bagong-normal-sa-edukasyon/

Umali, J. (2021). Masamang Epekto ng online learning. PhilStar. Masamang Epekto ng online learning Vercide, J. (2020). Online classes: Epektibo nga ba para matuloy ang pasukan. The Lookout. https://thelookout.com.ph/article/online-classes-epektibo-nga-ba-para-matuloy-ang-pasukan 
Gallega, M., Guyala, C., Formento, M. L., Gamba, A., \& Gelilio, E.

42 Consortia Academia Publishing (A partner of Network of Professional Researchers and Educators) 